\title{
Optimization of Air-cooling System for a Lithium-ion Battery Pack
}

\author{
Sungwook Jin ${ }^{1 *}$, Min-Sik Youn ${ }^{1}$, and Youn-Jea Kim² \\ ${ }^{1}$ Graduate School of Mechanical Engineering, Sungkyunkwan University, Korea \\ ${ }^{2}$ School of Mechanical Engineering, Sungkyunkwan University, Korea
}

\begin{abstract}
Lithium-ion batteries have been used as energy storage technologies for electric vehicles or power plants due to their high energy density, low self-discharge rate, and long lifespan. Since the temperature of the batteries are directly related with their durability, distributing the temperature uniformly and efficiently is critically important. In this study, a technology using forced convection with air was implemented to remove heat of the battery cells inside a package. The performance of the cooling system was evaluated by changing the gap distance between the battery cells and the configurations of the air channel. In order to improve the cooling performance of the battery, the shape of the battery module was optimized. To begin the optimization process, a sensitivity analysis was conducted to analyze the influence of the design parameters on the battery performance. Based on the result from the analysis, an optimization process was performed to determine an optimum channel design. As a result of the optimization, a battery cell package with the lowest maximum temperature and a minimum deviation between the temperature in between each cell was selected.
\end{abstract}

\section{Introduction}

Recently, electric vehicles(EVs) have been growing in popularity as eco-friendly transportation because they are free from environmental pollutants such as NOX and PM [1]. Also, as the energy density of secondary batteries increases, many research papers on the EVs that use secondary batteries as a source of energy are actively carried out. The most frequently installed secondary battery in EVs is a lithium-ion battery(LIB), which is a rechargeable battery consisting of an anode, cathode, separator, and electrolyte. A LIB is charged and discharged by the transfer of charge through redox reaction between an anode and cathode. LIB has a higher energy density than other secondary batteries, six times that of Lead Acid batteries and four times that of Nickel Cadmium batteries.

The battery system installed in an EV comprises several packs of modules with several cells inside each module. The battery cells inside the module are connected in a series with small gap between the cells to increase energy density. When the LIB is charged or discharged, heat is generated due to the Joule heating effect and reaction heat of the electrolyte [2]. However, the structure of a battery causes the heat to be isolated inside the module. When a battery cell is exposed to a high temperature environment, gas is generated inside the battery due to a chemical side reaction, the battery expands [3], and the internal resistance increases, thereby irreversibly decreasing the capacity [4]. Therefore, it is essential to maintain a temperature between $15^{\circ} \mathrm{C}$ and $35^{\circ} \mathrm{C}$ using a suitable cooling system for the best LIB performance [5].

There are water and air cooling methods to cool battery modules in the EVs. The water cooling method can remove more heat than the air cooling method. But the structure of the water cooling method is complex, and there is a risk that the coolant may leak. On the other hand, in the air cooling method, the cooling performance is relatively low due to the small heat capacity of the air. However, compared to the water cooling method, the air cooling method is easy to repair and has a simple design. In addition, since the air cooling method consumes less power, research on a battery system with the air cooling method is being actively conducted. Chen et al. [6] studied the cooling effect according to various patterns of internal flow by changing the position of the inlet and outlet of air in a battery cooling system. Zhang et al. [7] studied an air cooling battery system with a spoiler installed in the flow channel to increase the amount of air flowing between battery cells. Liu et al. [8] conducted a study on battery cooling systems that reduce the difference between the minimum and maximum temperatures of the battery cells by inducing a constant airflow inside the module using wedgeshaped plenums. Hong et al. [9] installed a secondary vent in the battery module plenum to reduce the temperature of the maximum temperature between the battery cells. Chen et al. [10] designed a fin shape that shows great cooling performance in the air cooling battery system to which multiple fins are applied using

\footnotetext{
*Corresponding author: jsw194@skku.edu
} 
multi-objective genetic algorithm (MOGA) optimization based on the surrogate model.

In this study, the shape of a battery module is optimized to achieve a maximum cooling efficiency of the air cooling system to ensure the best performance of the battery. The ratio of the air inlet, outlet angle, and the ratio of the gap between every other cell were selected as design variables. To validate numerical analysis, the temperature of the cell surface cooled by forced convection was compared with the experimental results of Xie et al. [11]. To perform the shape optimization of battery module, design points were constructed using the design of experiments (DOE), and a surrogate model was created using the results of the DOE. Based on the surrogate model, a Pareto set was determined using a MOGA based on the Nondominated Sorting Genetic Algorithm (NSGA-II). Finally, in the Pareto set, an optimized shape of the battery module was found for a minimum temperature when electric charges are discharged from the battery module to minimize the temperature deviation between cells.

\section{Model description}

\subsection{Reference model}

The battery module used in the study consists of a total of 10 battery cells, which are shown in Fig. 1 . The battery is prismatic cells type, and the size of a battery cell is $16 \mathrm{~mm} \times 65 \mathrm{~mm} \times 131 \mathrm{~mm}$ (length $\times$ width $\times$ height). Ten battery cells are connected in series and face each other with adjacent batteries. There is a passage through which air can pass between the battery cells, and the distance between each passage is $6 \mathrm{~mm}$. The battery module is $230 \mathrm{~mm} \times 73 \mathrm{~mm} \times 175 \mathrm{~mm}$, and the thickness of the plate surrounding the module is 2 $\mathrm{mm}$. At the bottom of the battery module, there is an inlet for air, and there is an outlet for air discharge in the upper part of the same direction.

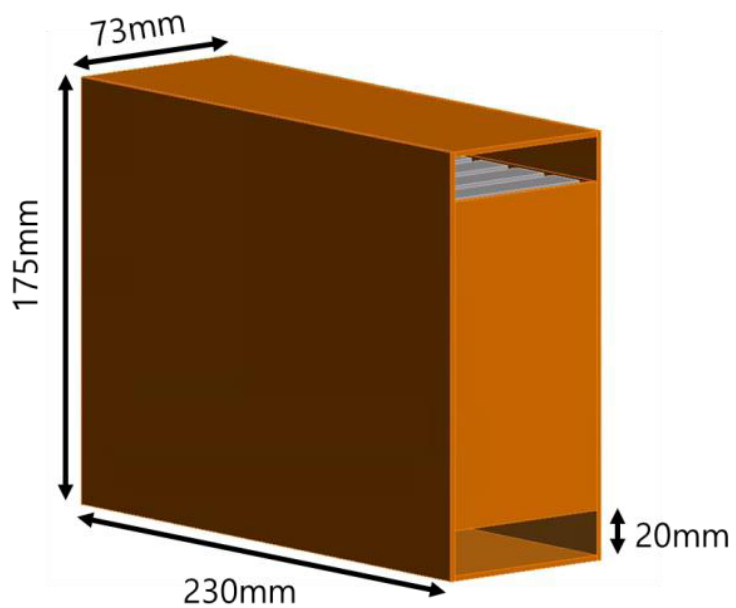

Fig. 1. 3D model of a battery module and cells

\subsection{Design variables}

The design variables of the battery module were selected as variables that can induce the flow inside the battery module. The angle of the air inlet channel $\left(\theta_{1}\right)$ and the angle of the air outlet channel $\left(\theta_{2}\right)$ were selected as design variables in order to induce air flow to improve heat transfer performance and to reduce temperature deviation of battery cells. In addition, in order to make the air flow through the passages between the battery cells uniform, the common difference of the width of the air passages between the battery cells was selected as a design variable.

The angle of the air inlet and outlet channels was changed from $0^{\circ}$ to $4^{\circ}$ based on the area where air enters and exits. The sum of the gaps between the 11 battery cells is always $66 \mathrm{~mm}$, and the following equations determine the gap between the battery cells.

$$
\begin{aligned}
& g_{n}=g_{1}+(n-1) d \\
& \sum_{i=1}^{11} g_{i}=66
\end{aligned}
$$

where $g_{n}$ is the gap of nth air passage, $\mathrm{d}$ is common difference.

The gap between the battery cells was adjusted to increase or decrease the gap based on the first battery by adjusting common difference in the range of -0.4 to 0.4 .

\section{Numerical solution}

\subsection{Computational domain and boundary conditions}

In this study, ten battery cells and cooling channels were constructed as analysis domains to calculate the cooling of the battery module numerically. The domains used in the numerical analysis were set as the fluid region and the solid region, the region where the cooling air moves as the fluid region, and the battery cell as

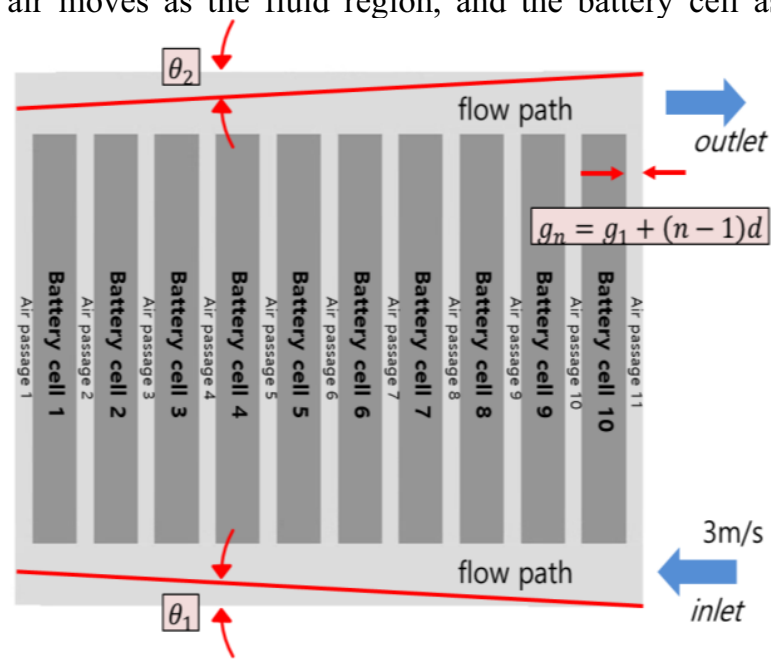

Fig. 2. Schematic of design variables in the battery module. 
the solid region. The inlet and outlet parts of the cooling air in the fluid region were composed of hexahedral mesh, and the region between battery cells was composed of tetrahedral mesh. The surface of the battery cell was composed of five layers of inflation layer in order to accurately calculate the convective heat transfer occurring on the surface, and $y+$ was configured to be less than 3 in all surface of battery cells. The solid region simulated 10 battery cells, and each battery cell was composed of a tetrahedral mesh. The battery cells are all connected in series, and each battery emits $3 \mathrm{~A}$ current. Heat generation conditions were set for each battery to simulate the heat source generated when current is discharged from the battery, and the heat generated by each battery is shown in the Table 1 .

In the case of the boundary conditions, the same conditions used in the experiment conducted by Xie et al. [11]. The working fluid used in the flow and heat transfer analysis was set to $298.15 \mathrm{~K}$ of air, and the average velocity of the inlet flow was $3 \mathrm{~m} / \mathrm{s}$. A pressure outlet condition was applied to the outlet. The properties of the battery cells in the solid area are indicated in Table 2 .

\subsection{Numerical method}

In this study, in order to analyze the flow and heat transfer characteristics of the fluid flow inside the battery module, the flow field is assumed to be a threedimensional, steady-state. ANSYS Fluent 20.0, a commercial computational fluid dynamics code, was used to solve the governing equations. The flow inside the battery module was calculated by the Reynolds Averaged Navier-Stokes (RANS) equation, and the pressure and velocity terms of the continuity equations are solved using a pressure-based coupled solver.

In order to select the turbulence modeling to be used in the analysis, the Reynolds number of the flow inside the module was calculated. The Reynolds number of cooling air entering the module inlet is about 7,700, and in general, when the Reynolds number in the internal flow reaches about 2,300, the fluid flow starts to transition from laminar flow to turbulent flow. Accordingly, numerical analysis was performed by judging the flow of this study as turbulent flow. In this study, the Realizable $\mathrm{k}-\varepsilon$ model, a turbulence model known to be well suited to the internal trapped flow where Reynolds stress is dominant, was used. The governing equations used in numerical analysis are as follows:

Continuity equation:

$$
\nabla \cdot(\rho \vec{v})=0
$$

Momentum equation:

$$
\frac{\partial}{\partial t}(\rho \vec{v})+\nabla \cdot(\rho \vec{v} \vec{v})=-\nabla p+\nabla \cdot(\overline{\bar{\tau}})+\rho \vec{g}
$$

where $p$ is the static pressure and $\overline{\bar{\tau}}$ is the stress tensor:

$$
\overline{\bar{\tau}}=\mu\left[\left(\nabla \vec{v}+\nabla \vec{v}^{T}\right)-\frac{2}{3} \nabla \cdot \vec{v} I\right]
$$

Table 1. Properties of the air and battery cells[11].

\begin{tabular}{ccccc}
\hline & $\begin{array}{c}\text { Density } \\
\left(\mathrm{kg} / \mathrm{m}^{3}\right)\end{array}$ & $\begin{array}{c}\text { Specific } \\
\text { heat } \\
(\mathrm{J} / \mathrm{kg} \cdot \mathrm{K})\end{array}$ & $\begin{array}{c}\text { Thermal } \\
\text { conductivity } \\
(\mathrm{W} / \mathrm{m} \cdot \mathrm{K})\end{array}$ & $\begin{array}{c}\text { Dynamic } \\
\text { visocity } \\
(\mathrm{kg} / \mathrm{m} \cdot \mathrm{s})\end{array}$ \\
\hline Air & 1.225 & 1006.43 & 0.0242 & $1.74 \times 10^{-5}$ \\
Battery & 1958.7 & 733 & 2.7 & - \\
\hline
\end{tabular}

Table 2. Heat generation rate of each batteries[11].

\begin{tabular}{cc}
\hline Battery number & Heat generation rate $(\mathrm{W} / \mathrm{m} 3)$ \\
\hline 1 & 20993 \\
2 & 22296 \\
3 & 22845 \\
4 & 22930 \\
5 & 23948 \\
6 & 29800 \\
7 & 32051 \\
8 & 33988 \\
9 & 35341 \\
10 & 35854 \\
\hline
\end{tabular}

where $\mu$ is the molecular viscosity and $\mathrm{I}$ is the unit tensor.

The following energy equation was used to calculate the convective heat transfer between the cooling fluid and the battery cell.

Energy equation:
$\begin{aligned} & \frac{\partial}{\partial t}(\rho E)+\nabla \cdot(\vec{v}(\rho E+p)) \\ & =\nabla \cdot\left(k_{e f f} \nabla T-\sum_{j} h_{j} \vec{J}_{j}+\left(\overline{\bar{T}}_{e f f} \cdot \vec{v}\right)\right)\end{aligned}$

\section{Optimization}

\subsection{Objective function}

In this study, two objective functions were defined to design a cooling system to maximize battery performance using forced convection cooling. First, the maximum temperature on the surface of 10 battery cells was selected as a function to cool the heat generated by the battery cells as much as possible. Secondly, the standard deviation of the highest temperature of each battery cell surface was selected as a function to minimize the temperature deviation between 10 battery cells. The purpose of the optimization problem in this study is to minimize the two objective functions and can be expressed as follows:

$$
\begin{array}{ll}
\text { Find } & \theta_{1}, \theta_{2}, d \\
\text { To minimize } & f_{1}, f_{2} \\
& 0 \leq \theta_{j} \leq 40, \quad j=1,2 \\
& -0.4 \leq d \leq 0.4
\end{array}
$$

where $f_{1}$ is maximum temperature of battery cells and $f_{2}$ is standard deviation of maximum temperature of each battery cells. 


\subsection{Design of experiment}

To make the surrogate model efficiently, design points were constructed using the design of experiment(DOE) method. The DOE is designed to obtain the maximum information with minimum effort and helps to find the minimum design points in designing a surrogate model [12]. In this study, design points were constructed using Latin Hypercube Sampling (LHS), one of the Space Filling Design methods of the DOE method. LHS distributes design factors evenly in Latin square and has the advantage of being able to extract more diverse design points by preventing overlapping design points. According to Wang et al. [13], the number of appropriate design points for designing surrogate models when using LHS is ten times the number of design factors, but in this work, 45 design points are constructed to design more accurate surrogate models.

\subsection{Surrogate model}

A surrogate model is a technique for approximately obtaining functions based on points calculated by the DOE. The approximation function was calculated by a quadratic polynomial of $x$ for each objective function $y$ based on the analysis results of the design point and can be expressed as follows:

$$
y=\beta_{0}+\sum_{i=1}^{N} \beta_{i} x_{i}+\sum_{i=1}^{N} \beta_{i i} x_{i}^{2}+\sum_{j=1}^{N} \sum_{\substack{i=1 \\ i<j}}^{N} \beta_{i j} x_{i} x_{j}
$$

where $N$ is the number of design variables and $\beta$ is an undefined coefficient.

\subsection{Multi-objective genetic algorithm}

The optimization algorithm for finding the optimal point of the objective function is Multi-Objective Genetic Algorithm (MOGA), one of the Non-dominated Sorted Genetic Algorithm-II (NSGA-II) methods. This technique is suitable for optimization problems with more than two objective functions and has the advantage of having a very high probability of finding a global optimum by searching the entire design domain [14]. The algorithm mimics the inheritance of an ecosystem, which uses selection, mutation and cross-over to produce new populations repeatedly and to find the optimal point of the solution [15]. The initial population size and population size per iteration of the genetic algorithm used in this study are set to 2000 and 100 , respectively. The genetic algorithm was repeated until the fitness value was less than 1 or the number of iterations reached 1000 .

\section{Result and discussion}

\subsection{Validation of CFD}

To validate the numerical analysis results, we compare

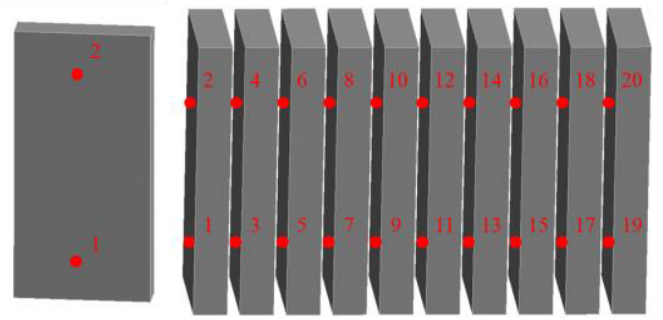

Fig. 3. Temperature measuring points of cells.

them with the experimental results of Xie et al. [11]. In the experiment, a temperature sensor was attached to two points on the front and bottom of each battery cell to measure the temperature of the battery cell surface due to the cooling of air when the current was emitted from the battery. A total of 20 temperature sensors were used, and the location of the temperature sensors is shown in Fig. 3. Numerical analysis also calculated the temperature at the location where the temperature sensor was attached to each battery cell. The area-weighted average of a quantity is computed by dividing the summation of the product of the selected field variable and facet area by the total area of the surface:

$$
\frac{\sum_{f}\left|\underline{A}_{f}\right| \varphi_{f}}{\sum_{f}\left|\underline{A}_{f}\right|} \frac{1}{A} \int \phi d A=\frac{1}{A} \sum_{i=1}^{n} \phi_{i}\left|A_{i}\right|
$$

Figure 4 shows experimental and numerical analysis results. In addition, the relative error of the experimental result and the relative error between the experimental result and the numerical analysis result was calculated at each measuring point. It shows similar trends in simulation and experimental results. The slight difference between experiments and simulations is that the heat generation of the battery cell is constant as set in the simulation, but the actual battery cell changes in heat generation over time as the current is released. However, the relative errors of the temperature at all of measuring points were less than $5 \%$, respectively. This indicates that the method presented in this paper was effective and feasible.

\subsection{Development of surrogate model}

Table 3 shows the 45 design points selected by DOE and the results at each design point. By substituting DOE results for polynomial regression, the objective functions $f_{1}$ and $f_{2}$ are calculated as second-order polynomials for $x_{1}, x_{2}$, and $x_{3}$. The calculated expression is the surrogate model, and the expressions for $f_{1}$ and $f_{2}$ are as follows:

$$
\begin{aligned}
f_{1}= & -0.0175 x_{1}^{2}+0.0387 x_{2}^{2}+4.9804 x_{3}^{2} \\
& -0.0312 x_{1} x_{2}+1.0771 x_{2} x_{3}-0.1827 x_{3} x_{1} \\
& +0.1705 x_{1}-0.1483 x_{2}-0.4261 x_{3} \\
& +314.8149 \\
f_{2}= & -0.0175 x_{1}^{2}+0.0387 x_{2}^{2}+4.9804 x_{3}^{2} \\
& -0.0312 x_{1} x_{2}+1.0771 x_{2} x_{3}-0.1827 x_{3} x_{1} \\
& +0.1705 x_{1}-0.1483 x_{2}-0.4261 x_{3} \\
& +314.8149
\end{aligned}
$$




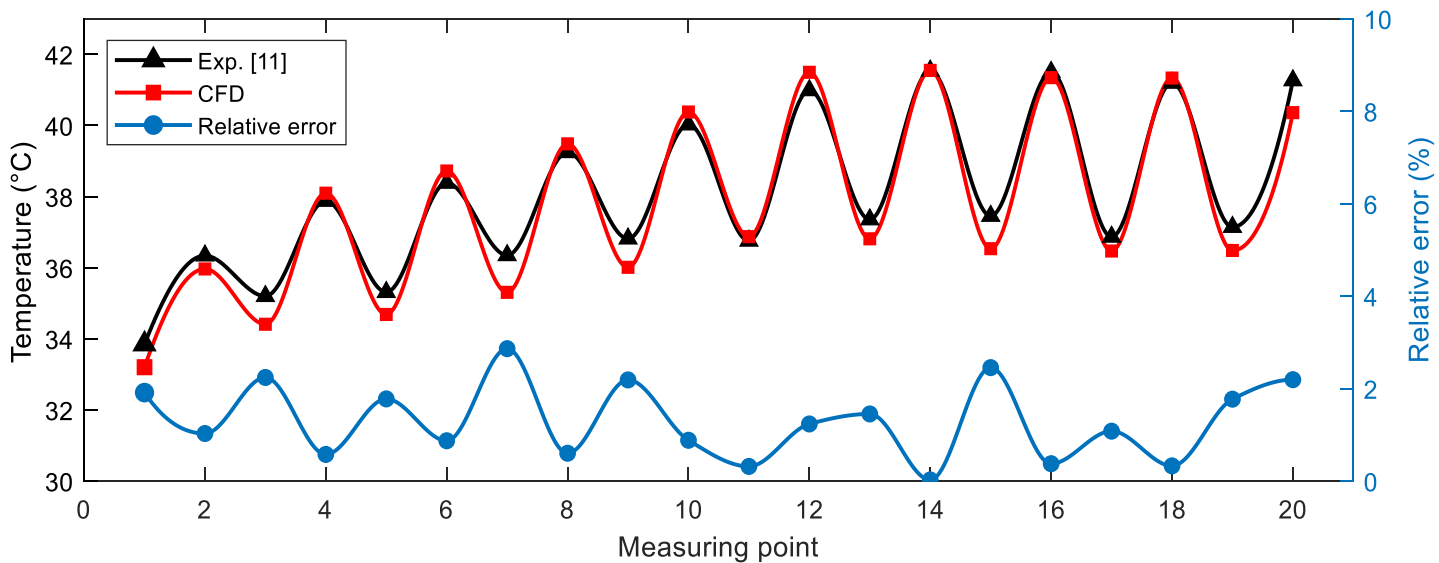

Fig. 4. The resulting plot of the experiment and analysis at the temperature measurement point and the error of the two results.

R-squared and Root Mean Squared Error (RMSE) were calculated to measure the accuracy of the surrogate model. R-squared represents the coefficient of how well the values fit compared to the original values. The value from 0 to 1 interpreted as percentages. The higher the value is, the better the model is:

$$
R^{2}=1-\frac{\sum\left(y_{i}-\hat{y}\right)^{2}}{\sum\left(y_{i}-\bar{y}\right)^{2}}
$$

where $\hat{y}$ is the predicted value of $y$ and $\bar{y}$ is the mean value of $y$.

RMSE is the error rate by the square root of the difference between the original and predicted values extracted by squared the average difference over the data set. RMSE is represented:

$$
R M S E=\sqrt{\frac{1}{N} \sum_{i=1}^{N}\left(y_{i}-\hat{y}\right)^{2}}
$$

To determine the accuracy of the surrogate model, R-squared and RMSE for $f_{1}$ and $f_{2}$ were calculated and presented in Table 4. From the Table 4, it is shown that the values of R-square of each parameters are close to 1 , furthermore the values of R-square of each parameters are close to 0 . From the results of R-squared and RMSE, it can be deduced that calculated surrogated model predict well the exact solution.

In addition, to determine how well the surrogate model predicted the actual value, it is shown in Fig. 5. In the plot, the square point represents the predicted value for the observed value, and the more it is placed diagonally, the more accurate the prediction is. As a result of Fig. 5, the surrogate model can be judged as a good predictor of the actual value because most of the points in both objective functions are distributed near the diagonal.
Table 3. Design points of DOE and CFD result at the

\begin{tabular}{|c|c|c|c|c|c|}
\hline $\begin{array}{c}\text { Design } \\
\text { point }\end{array}$ & $\begin{array}{c}\text { Inlet } \\
\text { angle } \\
\left(\theta_{1}\right)\left[^{\circ}\right]\end{array}$ & $\begin{array}{l}\text { Outlet } \\
\text { angle } \\
\left(\theta_{2}\right)\left[^{\circ}\right]\end{array}$ & $\begin{array}{l}\text { Diff. } \\
\text { (d) }\end{array}$ & $\begin{array}{c}\text { Max. } \\
\text { temp. } \\
\left(f_{1}\right)\left[{ }^{\circ} \mathrm{C}\right]\end{array}$ & $\begin{array}{l}\text { Stan. } \\
\text { dev. } \\
{[K]}\end{array}$ \\
\hline 1 & 1.530 & 2.310 & -0.036 & 41.673 & 1.371 \\
\hline 2 & 2.310 & 3.177 & 0.356 & 43.024 & 1.420 \\
\hline 3 & 0.143 & 2.137 & -0.142 & 41.453 & 1.431 \\
\hline 4 & 1.877 & 0.577 & -0.178 & 41.982 & 2.147 \\
\hline 5 & 0.317 & 0.143 & -0.107 & 41.717 & 1.934 \\
\hline 6 & 3.697 & 0.403 & 0.107 & 41.967 & 1.393 \\
\hline 7 & 1.790 & 2.657 & -0.267 & 41.359 & 1.581 \\
\hline 8 & 3.610 & 3.263 & 0.000 & 41.613 & 0.933 \\
\hline 9 & 0.837 & 2.397 & 0.249 & 42.383 & 1.099 \\
\hline 10 & 0.577 & 1.010 & 0.160 & 41.855 & 1.268 \\
\hline & & & & & \\
\hline 36 & 0.923 & 3.437 & 0.142 & 42.244 & 1.036 \\
\hline 37 & 3.523 & 3.003 & 0.124 & 41.932 & 0.980 \\
\hline 38 & 3.437 & 1.703 & -0.284 & 41.789 & 1.960 \\
\hline 39 & 2.050 & 2.830 & -0.373 & 41.512 & 1.805 \\
\hline 40 & 3.957 & 3.090 & 0.036 & 41.706 & 0.876 \\
\hline 41 & 1.963 & 0.490 & -0.071 & 41.880 & 1.920 \\
\hline 42 & 1.703 & 2.223 & -0.356 & 41.733 & 1.992 \\
\hline 43 & 2.570 & 1.097 & 0.089 & 41.928 & 1.477 \\
\hline 44 & 0.750 & 0.317 & 0.267 & 42.086 & 1.135 \\
\hline 45 & 2.830 & 3.697 & 0.178 & 42.347 & 1.082 \\
\hline
\end{tabular}
design points

Table 4. R-Squared and RMSE result of output parameter

\begin{tabular}{ccc}
\hline & \multicolumn{2}{c}{ Output parameter } \\
\cline { 2 - 3 } & $\begin{array}{c}\text { Maximum } \\
\text { Temperature }\end{array}$ & Standard deviation \\
\hline $\mathrm{R}^{2}$ & 0.9519 & 0.9803 \\
$\mathrm{RMSE}$ & 0.1114 & 0.0649 \\
\hline
\end{tabular}




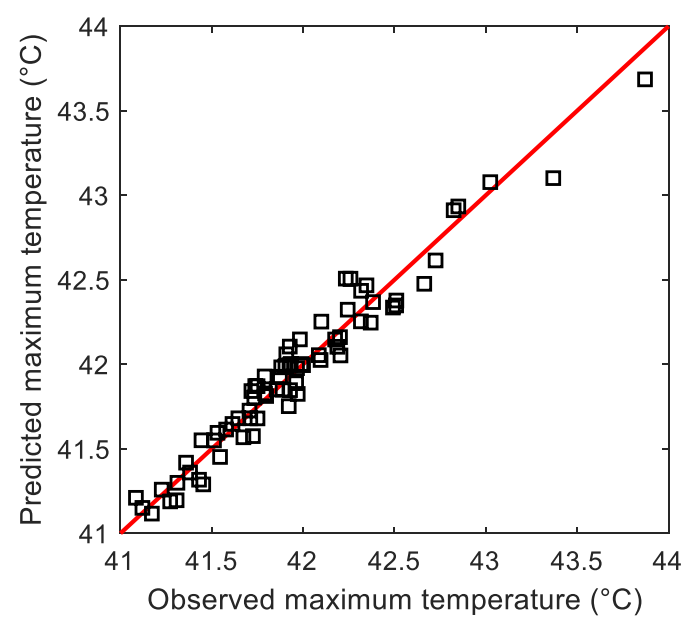

(a) Maximum temperature

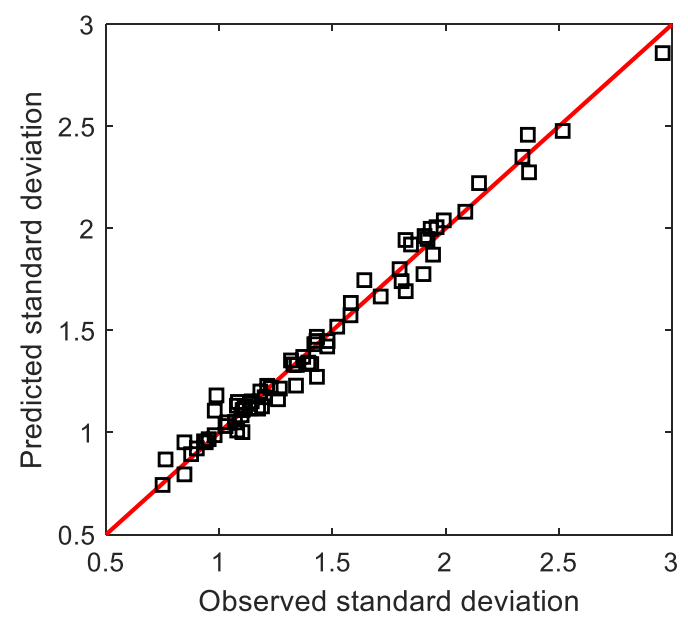

(b) Standard deviation

Fig. 5. Comparison of the predicted value by surrogate model with observed value.

\subsection{Sensitivity analysis}

Figure 6 indicates the sensitivity of the response variable to changes in the design variable by the response surface method. The $\mathrm{x}$-axis is a normalized number from 0 to 1 in the design range of the input variable. First, the response surface results for the maximum temperature of the battery cell showed that for $x_{1}$, it was increasing in the initial region of the design domain and then converging steadily. On the other hand, $x_{2}$ showed a minimum point near 0.6 in the design domain, and $x_{3}$ also showed a minimum point near 0.3 . As a result of the second output function, the standard deviation of the temperature, for $x_{1}$, the output function tends to be maximum near $0.55 . x_{2}$ showed a negative relationship in which the output function decreased as the value of the design variable grew, and $x_{3}$ showed that the minimum point of the output function was present near 0.8 . Results from sensitivity analysis show that the three input variables selected in this study have a significant impact on the two output variables, and that optimal points exist through the interaction between each variable.

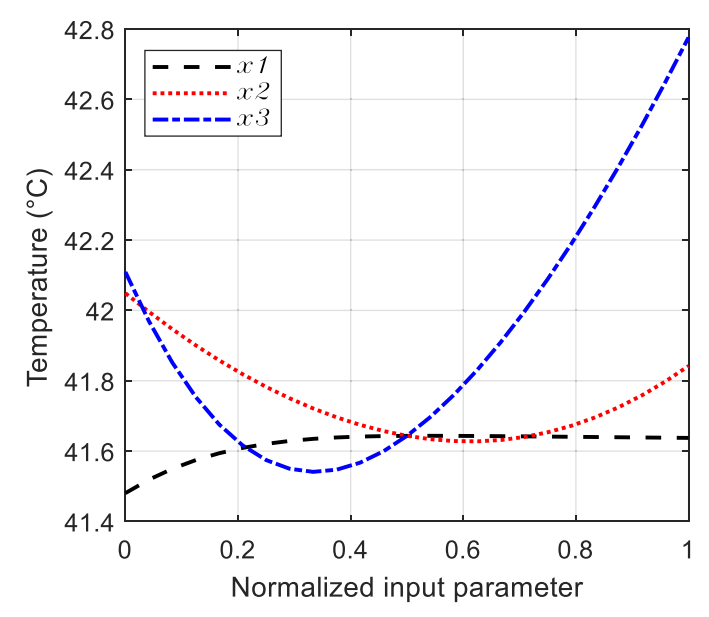

(a) Sensitivity of maximum temperature

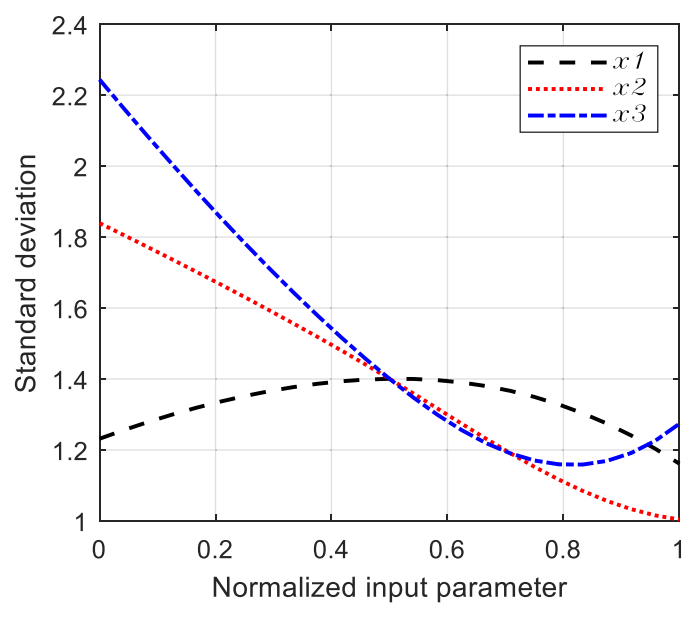

(b) Sensitivity of standard deviation of temperature

Fig. 6. Result of sensitivity with various design parameters.

\subsection{Results of MOGA}

The order of solution in the optimization problem depends on the number of objective functions. In multiple objective functions, the optimization problem has multiple solutions, which are called Pareto solutions. Among the Pareto solutions, two objective functions exhibit trade-off relationships, and the set that exists near the boundary of the solution set is called a Pareto front, and the solutions in this set are the optimal points for multi-objective optimization. Also, solutions that exist near the Pareto front and in the feasible area are called dominated solutions. Figure 7 represents Pareto solutions derived from this study. The red triangle represents the Pareto front and the surrounding points represent the dominated solution. We selected the solution at the bottom left of the Pareto front as the optimal point and applied it as the final optimal model in this study. The solution present at the bottom left of the Pareto front is selected as the optimal point, where the optimal shape is shown in Fig. 8. The optimal model has an inlet angle of $3.994^{\circ}$, an outlet angle of $3.995^{\circ}$, and a common differential between battery cells of 0.185 . The channel width between the inlet and outlet 


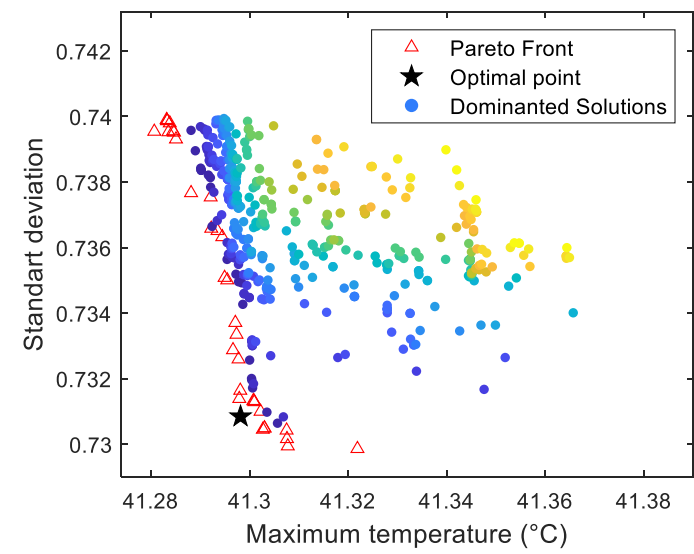

Fig. 7. Pareto solution.

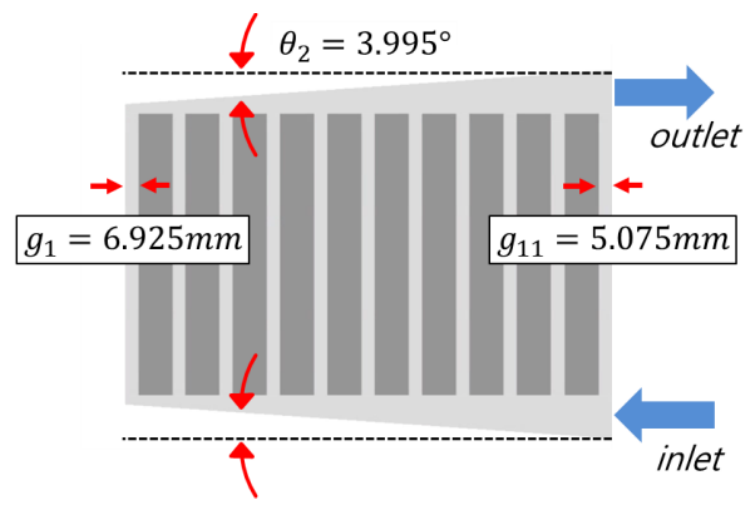

Fig. 8. Optimal design of battery module.

becomes narrower as the first battery approaches, but the gap between the battery cells widen. The optimization results and CFD results at the optimal point are shown in Table 5 and compared with the base model. The optimization results of MOGA and the results of CFD showed an error of $0.07 \%$ on the first objective function and $3.87 \%$ on the second objective function, which is judged to have made accurate predictions. The performance improvement by optimization showed a $1.202^{\circ} \mathrm{C}$ decrease in the maxim um temperature in the battery cell, and a $0.883 \mathrm{~K}$ decrease in the maximum temperature deviation between the battery cells from $1.632 \mathrm{~K}$ to $0.749 \mathrm{~K}$. Figure 9 shows the temperature distribution of battery cells in the underlying model and the optimal model. The results of the temperature distribution show that the optimal model shows a decrease in temperature where the heat is concentrated.

Table 5. Results of MOGA.

\begin{tabular}{c|c|c}
\hline & $\begin{array}{c}\text { Maximum } \\
\text { temperature }\left[{ }^{\circ} \mathrm{C}\right]\end{array}$ & $\begin{array}{c}\text { Temperature } \\
\text { standard } \\
\text { deviation }[\mathrm{K}]\end{array}$ \\
\hline Reference model & 43.529 & 1.632 \\
\hline $\begin{array}{c}\text { Optimal design } \\
\text { (pareto solution) }\end{array}$ & 42.297 & 0.720 \\
\hline $\begin{array}{c}\text { Optimal design } \\
\text { (CFD result) }\end{array}$ & 42.327 & 0.749 \\
\hline Relative error $(\%)$ & 0.07 & 3.87 \\
\hline
\end{tabular}

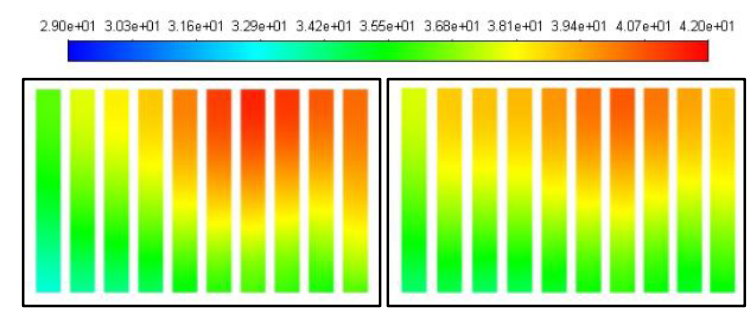

$\begin{array}{ll}\text { (a) Reference model } & \text { (b) Optimal model }\end{array}$

Fig. 9. Comparison of battery cells temperature distribution between the reference and optimal models

\section{Conclusion}

In this study, we perform a shape optimization for air cooling battery system. In the optimal design process, the surrogate model was created using the DOE, and the correlation between a number of design variables and output variables was analyzed through sensitivity analysis. As a result of the shape optimization, the optimal model reduces the maximum temperature of the battery cell by $1.202^{\circ} \mathrm{C}$ compared to the reference model, and the deviation of the maximum temperature of the battery cell by $0.883 \mathrm{~K}$.

\section{References}

1. J. M. Tarascon, and M. Armand, Materials for sustainable energy: a collection of peer-reviewed research and review articles from Nature Publishing Group, 171 (2011)

2. S. C. Chen, C. C. Wan and Y. Y. Wang, Journal of power sources, 140(1), 111 (2005)

3. J. B. Siegel, A. G. Stefanopoulou, P. Hagans, Y. Ding, and D. Gorsich Journal of the Electrochemical Society, 160(8), A1031 (2013)

4. E. Redondo-Iglesias, P. Venet, and S. Pelissier, Measuring reversible and irreversible capacity losses on lithium-ion batteries in Proceedings of the IEEE Vehicle Power and Propulsion Conference VPPC, 17-20 Oct. 2016, Hangzhou, China (2016)

5. S. Ma, M. Jiang, P. Tao, C. Song, J. Wu, J. Wang and W. Shang, Progress in Natural Science: Materials International, 28(6), 653 (2018)

6. K. Chen, W. Wu, F. Yuan, L. Chen and S. Wang Energy, 167, 781 (2019)

7. F. Zhang, A. Lin, P. Wang and P. Liu, Applied Thermal Engineering, 182, 116062 (2021)

8. Z. Liu, Y. Wang, J. Zhang and Z. Liu, Applied thermal engineering, 66(1-2), 445 (2014)

9. S. Hong, X. Zhang, K. Chen and S. Wang, International Journal of Heat and Mass Transfer, 116, 1204 (2018) 
10. L. Cheng, A. Garg, A. K. Jishnu and L. Gao, Journal of Energy Storage, 31, 101645 (2020)

11. J. Xie, Z. Ge, M. Zang and S. Wang, Applied Thermal Engineering, 126, 583 (2017)

12. H-S. Kang and Y-J. Kim, International Journal of Fluid Machinery and Systems, 9(2), 143 (2016)

13. L. Wang and D. Beeson, International Design

Engineering Technical Conferences and Computers and Information in Engineering Conference, 49026 (2019)

14. S. Sanaye and H. Hajabdollahi, International Journal of Thermal Sciences, 48(10), 1967 (2009)

15. R. V. Rao, A. Saroj, P. Ocloń, J. Taler and D.

Taler, Heat Transfer Engineering, 39(13-14), 1201 (2018) 